\title{
Abiezione nell'arte postmoderna come risultato di una cultura post-traumatica
}

\author{
Amparo Latorre Romero \\ Università degli Studi di Roma La Sapienza \\ amparometrallas@gmail.com
}

RESUMEN: Obiettivo di questo articolo è identificare gli elementi che permettono di costruire un punto di vista storico-artistico e critico a partire dall'Olocausto fino ad arrivare alla questione dell'abietto. Se il concetto di abietto è stato, nel corso del Novecento, sia nella storia dell'arte che nell'estetica o nella semiotica, alla base del superamento dei limiti simbolici, psichici e culturali, per tentare di trasgredire o di perturbare i sistemi identitari sia individuali che collettivi, nell'abiezione nell'arte tali questioni si presentano in form estremamente originali. Lo studio cerca dunque di dimostrare, anche attraverso una linea teorica che Julia Kristeva ha trattato nel suo libro Pouvoirs de l'horreur, che il discorso si costruisce su concetti e poetiche connessi all'abiezione. Partendo da queste considerazioni sarà interessante riconoscere la presenza di immagini dell'abietto.

PALABRAS CLAVE: Abietto; Trauma; Identità; Arte Postmoderna.

\section{Abjection in Post Modern Art as a Result of Post Traumatic Culture}

ABSTRACT: The aim of this paper is to identify the elements that allow us to build a theoretical, art historical and critical view from the Holocaust to the matter of abjection. If the concept of abjection has existed during the twentieth century, not only in art history, but also in aesthetics or semiotics, as a base for overcoming symbolic, psychic and cultural limitations in order to transgress or perturb the individual and collective identity system, in abjection art work these questions are presented in extremely original images. The purpose of the essay is to try to demonstrate, from a theoretical perspective, following Julia Kristeva and her book Pouvoirs de l'horreur, that the discussion is built on concepts or poetics connected to abjection. Using these considerations to approach the question, it will be interesting to identify the presence of abject images.

KEYWORDS: Abjection; Trauma; Identity; Postmodern Art.

Recibido: 13 de abril de 2017 / Aceptado: 13 de julio de 2017.

\section{Perché l'abiezione?}

Questo articolo ha l'obiettivo di dimostrare come l'abiezione segua dei passaggi fondamentali: attraversare il trauma, sublimarlo e farsi arte. Detto altrimenti l'abiezione è indispensabile in quanto, se il corpo non espelle i rifiuti, "cade» e si trasforma in cadavere. Innanzitutto dobbiamo ricordare che l'abiezione è un concetto che Julia Kristeva ci spiega nel suo saggio Poteri dell'orrore apparso nel 1980. Citiamo la descrizione di Kristeva, in cui l'abietto è quello che:

perturba una identità, un senso, un ordine. Quello che non rispetta i limiti, i posti, le regole. La complicità, l' ambiguo, il misto. II traditore, il bugiardo, il criminale con la coscienza pulita, lo stupratore svergognato, l' assassino che pretende di salvare [...]. Ogni crimine in quanto segnala la fragilità della legge è abietto, ma il crimine premeditato, l'assassinio subdolo, la vendetta ipocrita lo sono ancora di

Cómo citar este artículo: LATORRE ROMERO, Amparo, "Abiezione nell'arte postmoderna come risultato di una cultura post-traumatica», Boletín de Arte-UMA, n. 38 , Departamento de Historia del Arte, Universidad de Málaga, 2017, pp. 109-116, ISSN: 0211-8483 , DOI: http://dx.doi.org/10.24310/BoLArte.2017.v0i38.3364 
più perchè aumentano l'esibizione della fragilità legale. Colui che rifiuta la morale non è abietto, si può avere grandezza nell' amorale e perfino in un crimine ribelle che ostenta un'irriverenza verso la legge. L'abiezione invece è immorale, tenebrosa, losca, torbida [...] (Kristeva,1980: 6).

\section{Trauma}

Basterebbe interrogarsi sul concetto di trauma; etimologica-

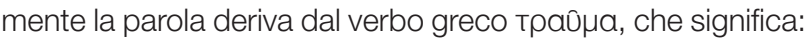
rovinare, danneggiare, perforare. II trauma, per sua stessa natura, ci impedisce di dare un significato e, quindi, di trovare una spiegazione razionale ad un' esperienza vissuta da un individuo nel corso della sua vita. II trauma stabilisce in un certo senso un contatto tra il soggetto coinvolto e la realtà della morte. II concetto di trauma, in quanto fa riferimento ad un' esperienza che interrompe di fatto l'andamento normale della vita di un individuo, ha una sua complessità e, per essere elaborato, richiede uno sforzo cognitivo e un lavoro che chiama in causa le nostre emozioni.

Concetto profondamente ed ampiamente studiato nel libro di Carla Subrizi, Azioni che cambiano il mondo. Donne, arte e politiche dello sguardo. Specialmente nel capitolo l'arte incontra il trauma, citando il testo:

Lo sguardo è «toccato" e con ciò si presenta come uno sguardo sensibile o, come dice Ettinger, è la visione che si manifesta come un campo psichico. È nell'arte, come hanno dimostrato le pratiche artistiche nella seconda metà del XX secolo, soprattutto nelle forme che le artiste hanno introdotto o concepito, che secondo Ettinger avviene il passaggio dal «fantasma al trauma». L'arte incontra la psicoanalisi e scava nel linguaggio, in molteplici direzioni: per capire, indagare e interrogare la psiche e il corpo, l'immaginazione e gli immaginari, l'intimità e l'ideologia, ciò che in essi si è stratificato e ciò di cui sono la premessa. II «bello», al quale proprio l'arte permette di accedere, in un modo diverso da quelle che sono state nella storia le definizioni di bello e bellezza nell'arte... (Subrizi,2012: 31-32).

Dobbiamo ritornare ancora a questo testo per trovare risposta alla domanda perché il corpo entra in azione. Come sottolinea Subrizi magistralmente nel capitolo Passioni e emozioni nell'arte della seconda metà del Novecento (Trauma e esperienza):

Ma il problema, sì il problema, che pone il XX secolo e soprattutto la seconda metà del secolo, al di là della Seconda guerra mondiale, è nel fatto che il corpo entra direttamente in azione. ॥ corpo diventa pratica significante ovvero produce e agisce processi di significazione. Questo non vuol dire che l'immagine o i processi siano venuti meno. Infatti considerare il corpo soltanto come entità che agisce fisicamente è forse, alla luce di quanto è poi emerso, anche nell'arte riduttivo. II corpo è anche traccia, impronta, memoria e immaginazione, e qui il ruolo delle emozioni, lo stato d'animo e l'affettività, si rivelano quali potenzialità costruttive di pensiero e azione. (Subrizi, 2012: 49).

Griselda Pollock nel suo libro After-Affect, After-Images: Trauma and Aesthetic Transformation in the Virtual Feminist Museum, afferma che esiste la possibilità che l'opera d'arte porti ad una riflessione, in modo particolare se si verifica un incontro tra il linguaggio estetico, nell'ottica del pensiero teorico-femminista, il trauma personale o collettivo (Pollock, 2013: 5-11). Alla base delle riflessioni di Pollock c'è il pensiero che tutti condividiamo uno stesso trauma, ossia la separazione alla nascita.

Jill Bennett, nel suo testo Empathic Vision Affect, Trauma and Contemporary Art, studia la questione dalla dialettica tra affetto, emozione e rappresentazione, legando fin dall'inizio questi concetti al lavoro sul trauma e sulla memoria (Bennett, 2005: 110). L'interesse dell'autore quindi si concentra in tutte quelle immagini in cui l'esperienza affettiva viene in qualche modo riattivata, consentendo la nascita di un nuovo linguaggio in grado di dare corpo all'opera e allo stesso tempo di prendere le mosse dall'esperienza vissuta.

L'artista, psicoanalista e teorica femminista Bracha Ettinger, figlia di sopravvissuti all'olocausto, ci offre un' interpretazione tra la soggettività e l'alterità introducendo, con la sua teoria matrixiale, una nuova dimensione simbolica secondo la quale si può riconoscere l'Altro nella propria diversità, e si può creare quindi una possibilità di incontro. Ancora l'artista ci fa riflettere, nel suo testo Regard et Espace-de-bord matrixiels (Ettinger, 1999) circa l'esistenza di un altro sguardo: oltre a quello puramente visivo, infatti, secondo l'artista esiste un' altra forma di sguardo, che riguarda le emozioni e gli affetti. II concetto matrixiale (il termine «matrix» 
deriva dal latino mater, utero), che si basa su una differenza femminile indefinita, laddove matrix per l'artista è innanzitutto un simbolo della relazione di alterità in cui si apre la possibilità di riconoscere l'Altro nella propria diversità, ossia una possibilità di incontro, crea un nesso profondo tra la nostra parte razionale e la nostra parte emotiva, che ci consente di oltrepassare i confini del dolore nell'incontro con l'altro. Bisogna mettere a fuoco che, come precetto teorico, il concetto di matrix è costruito in seno alla donna incinta e al suo rapporto con il feto, massima rappresentazione dell' abiezione.

Tornando al concetto di trauma possiamo dire che è stato affrontato per lo più negli anni Ottanta e Novanta del XX secolo, in una serie di studi noti come trauma studies, in cui si analizza la possibilità di rappresentazione del trauma e soprattutto il rapporto tra arte, trauma e psicoanalisi. Alcuni dei principali sostenitori di questo approccio teorico sono Cathy Caruth, Dominick La Capra e Maria Walsh. Per approfondire il rapporto tra arte e psicoanalisi e quello che lei definisce per «oggetto» anche presente nel discorso di Julia Kristeva come «oggetto abietto» è chiave il testo Art and psycoanalisis. Senza dimenticare il testo della semiotica Patrizia Violi, Paesaggi della memoria: II trauma, lo spazio, la storia Violi, 2014). Nel libro l'autrice ci fa riflettere sui luoghi in cui si sono consumati orrori e violenze di massa, invitando il lettore a riflettere su come dare un senso all'evento più traumatico del nostro secolo, la Shoa.

Nel saggio Heidegger e gli ebrei, la riflessione lyotardiana ci porta a considerare che dopo l'Olocausto rimane soltanto il trauma, condannando inoltre tutte quelle rappresentazioni create per far dimenticare.

A questo punto dobbiamo segnalare al lettore il riferimento al testo di TzvetanTodorov, Di fronte all'estremo:

È un fatto incontestabile. Essendo altresì un'affermazione di valori, l'opera d'arte testimonia un impegno morale e politico, e la scelta di tali valori può essere imputata solo all'artista. Di per sé i fatti non contengono lezioni, non sono trasparenti quanto il loro significato: è l'interpretazione che ne dà l'autore ad essere responsabile dei giudizi contenuti nell'opera.

Ai miei occhi, Shoah diventa problematico proprio in questo senso, e cioè non come opera d'arte, ma come tentativo di dire la verità su un certo mondo, volendone trarre un insegnamento (Todorov, 1992: 263).
Ricordando le parole di Jean-Luc Nancy, non basta condannare, bisogna mettere in luce le radici e questo può significare solo intervenire al cuore della nostra cultura.

L'umanità in tempi bui (Arendt, 2006) è una situazione passata e presente, sempre d'attualità; proviene chiaramente, come non poteva essere altro, dal testo di Hannah Aren$\mathrm{dt}$, e fa riferimento alla nostra condizione di sopravvissuti ad una condizione culturale post-traumatica o traumatica. Basta pensare che nel 2017 in Russia è stata depenalizzata la violenza sulle donne. Ci viene in mente subito un paragrafo del testo di Georges Didi-Huberman:

Le SS sapevano bene che una sola parola di un sopravvissuto del Sonderkommando avrebbe reso vani tutti i denieghi, tutti i ricami successivi sul grande massacro degli ebrei in Europa (Huberman, 2005: 16).

Appena affrontando tale discorso e facendoci le domande giuste ci accorgiamo che l'abiezione è reale e necessaria.

Non si può negare che il tema dell'abietto risulti scomodo e, perciò, spesso neutralizzato, in quanto stabilire le modalità con cui applicare un giudizio di gusto estetico non è semplice. Per questo, per poter distinguere l'abietto, dobbiamo prima definire la cornice etica e morale nella quale ci muoviamo.

\section{Pensiero Culturale}

È molto importante studiare il pensiero culturale e come questo gestisca l'asse paragdimatico etico-estetico e morale del corpo abietto. È inoltre importante ricordare ed accettare che l'essere umano, secondo le teorie dei filosofi classici, è uscito tra melma. Come ci ricorda Dante nel Canto Diciottesimo dell'Inferno della Divina Comedia:

\footnotetext{
Lo fondo è cupo sí, che non ci basta luogo a veder sanza montare al dosso de l'arco ove lo scoglio più sovrasta. Quivi venimmo; e quindi giù nel fosso vidi gente attuffata in uno sterco che da li uman privadi parea mosso. E mentre chío là giú con l'occhio cerco, vidi un col capo sí di merda lordo, Che non parea s'era laico o cherco.
} 


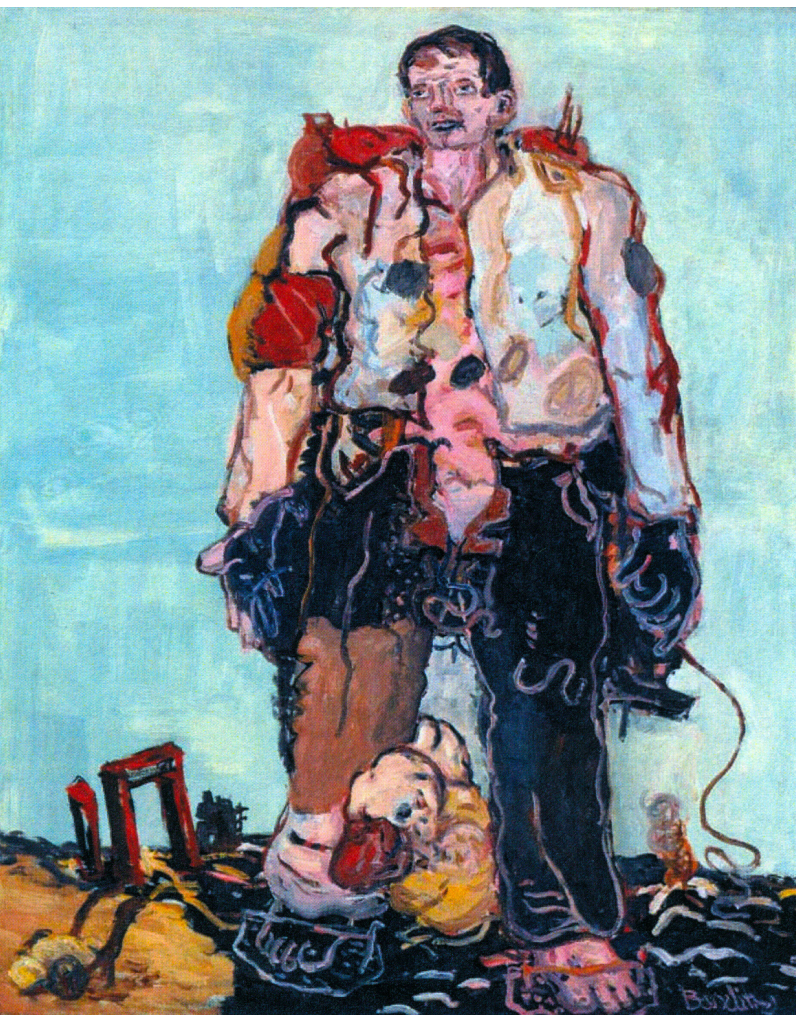

1. Georg Baselitz, Der Hirte (il pastore),1966. Olio su tela, $163 \times 130,7$ cm, Museum Frieder, Burda, Baden-Baden, Germania

Nel passo successivo Dante lascia gli adulatori sommersi nei loro escrementi e ritorna alla superficie del mondo per continuare a vedere le cose belle del cielo. L'essere umano, per sua stessa natura, non sopporta la nascita dell'informe, i rifiuti o la decomposizione della materia o della forma. Analizzando il discorso da un punto di vista antropologico, possiamo ricordare quando i nazisti avevano l'intenzione di far sparire milioni di persone a Treblinka, centro di sterminio nella parte orientale della Polonia, in un tempo assai breve, dal luglio del 1942 fino al novembre del 1943, durante la Seconda Guerra Mondiale. I nazisti facevano sparire i cadaveri bruciandoli, dicendo ai prigionieri che non erano corpi umani, ma solo escrementi, feci, rifiuti, merda. In concreto utilizzavano la parola dreck (dal tedesco merda).

Riconoscere che questo fatto è successo veramente e appartiene ormai alla memoria collettiva di tutti noi non è facile, per questo è importante che l'arte continui a rendere manifesto il vero volto della realtà stimolando la nostra rifles- sione. Come ci spiega Hannah Arendt nel saggio La banalità del male (Arendt, 1964) le azioni che si verificarono durante l'Olocausto erano mostruose, soprattutto perché venivano portate avanti da persone pressoché normali, da non considerarsi né demoniache né mostruose; era una questione di sopravvivenza.

È la stessa tesi che si sostiene con l'abiezione: affinché l'essere umano possa sopravvivere, deve contenere ed accettare la sua parte di abiezione. Questo discorso è necessario per testimoniare che l'abiezione appartiene in maniera profonda all' essere umano, per questo se ne deve parlare, per non rischiare di cadere nello sbaglio dell' occultazione.

Delle volte, come ci ricorda Hannah Arendt, la funzione di paria è quella che permette di esprimere in modo più libero la creatività; citando ancora la nostra filosofa:

Come spesso avviene nelle polemiche violente o appassionate, gli interessi meschini di certo gruppi, la cui eccitazione è esclusivamente dovuta a motivi concreti e che perciò cercano di travisare i fatti, si sono mischiati ben presto, in maniera inestricabile, ai ragionamenti ispirati di intellettuali che, al contrario, non si interessano minimamente dei fatti e li considerano soltanto un trampolino per lanciare «idee» (Arendt, 1964: 289).

Vogliamo fare riferimento ad un altro testo di Arendt, che sembra avere il titolo più attuale e conciso che ci possa essere: L'umanità in tempi bui.

II mondo diventa inumano, inospitale per i bisogni umani che sono bisogni umani -che sono bisogni di esseri mortali- quando è violentemente trascinato in un movimento in cui non si dà più alcun tipo di permanenza (Arendt, 2006: 56).

L'autoanalisi e l'autori flessione sono tendenze che domineranno tutto il Novecento, ed è importante che l'uomo, per comprendere profondamente la propria natura, accetti che l'abiezione è intrinseca all' esistenza umana.

\section{Analisi dell'abietto nell'arte Postmoderna}

Un esempio lo troviamo nella opera di Georg Baselitz, Der Hirte (il pastore),1966 [1]. Baselitz rappresenta una figura maschile con gli abiti confusi tra le macerie come un soldato 
sconfitto ritornato dalla guerra in una patria devastata, senza identità. L' artista ci mostra un paesaggio bruciato,il cui territorio devastato rappresenta la distruzione della guerra. Un' anatra bianca attaccata alla gamba sinistra del pastore, un resto bucolico delle anatre delle pitture precedenti dall'artista. L' immagine mostra la distruzione e la sopravvivenza mistificata a quelli che sono i nuovi eroi: il poeta, il pittore, un' arte realista socialista di una Germania appena uscita dalla guerra, frammentata ed sterile. II cromatismo dell'opera è basato sui complementari, si apprezza uno sfondo blu con molta saturazione di bianco. La figura maschile principale ha un contorno non sempre dallo stesso colore; a tratti diventa rosso, nel pantalone e nelle mani appare un contorno di bianco in diverse tonalità di rosso. Si osserva che i vestiti sono rimasti stracci, che non riescono a coprire totalmente il corpo dal pastore.

Un altro esempio lo troviamo nell'opera di Jake and Dinos Chapman, Great deads against the Dead, 1994 [2]. L'opera è una riflessione dell' incisione dei Disastri della guerra di Goya. Nell'acquaforte, da cui prende ispirazione l'installazione, tre figure si trovano impiccate su un tronco d'albero, assassinate e mutilate. Le vittime e le mutilazioni non finirono con la guerra di Indipendenza. In quest'opera la riflessione riguarda le modalità con cui facciamo fronte ai traumi della guerra e alla violenza; siamo originali quando ci tocca parlare di tortura? In questa installazione si riflette su come utilizziamo il corpo dell'orrore e della morte. I corpi sono dipinti dal colore della carnagione umana, anche con l'utilizzo dal rosso come segno delle parte strappate e si hanno utilizzato capelli umani per fare più reale l'opera.

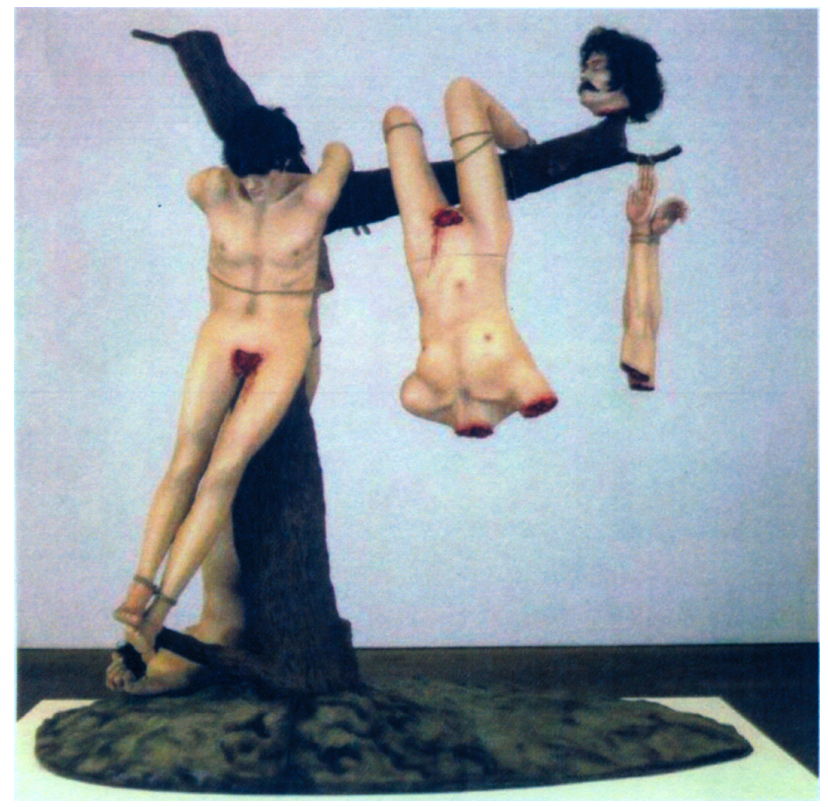

2. Jake and Dinos Chapman. Great deads against the Dead, 1994. Installazione, 277 × 244 x 152 cm. Saatchi Gallery, Londra

Gli artisti Gilbert \& George, con l' opera Naked Shit, 1994 [3], hanno ripresentato i rifiuti, le feci ed il corpo nella sua nudità. I britannici fanno riflettere su un corpo che lavora e non può e non deve essere punito per defecare. Gli artisti stano nudi senza i suoi abiti firmati. I corpi hanno la stessa grandezza che le feci. In quest'opera si cerca di equiparare il corpo umano vivo con quello abietto, la materia di rifiuto alla quale si ritornerà con la morte.

3. Gilbert and George. Naked shit, 1994. Fotografia, 253 × $639 \mathrm{~cm}$. Museo d'Arte Contemporanea Donna Regina (M.A.D.R.E), Napoli

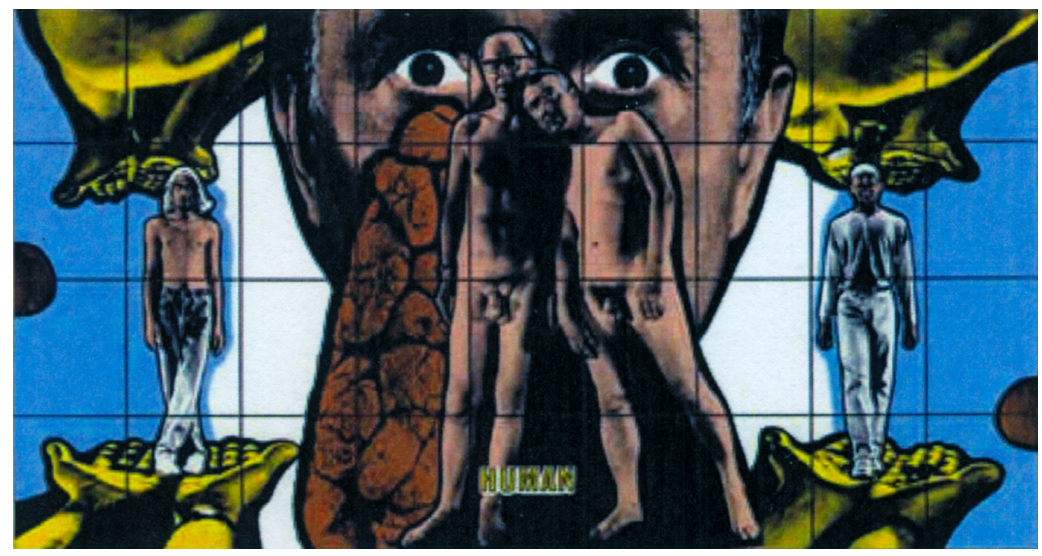




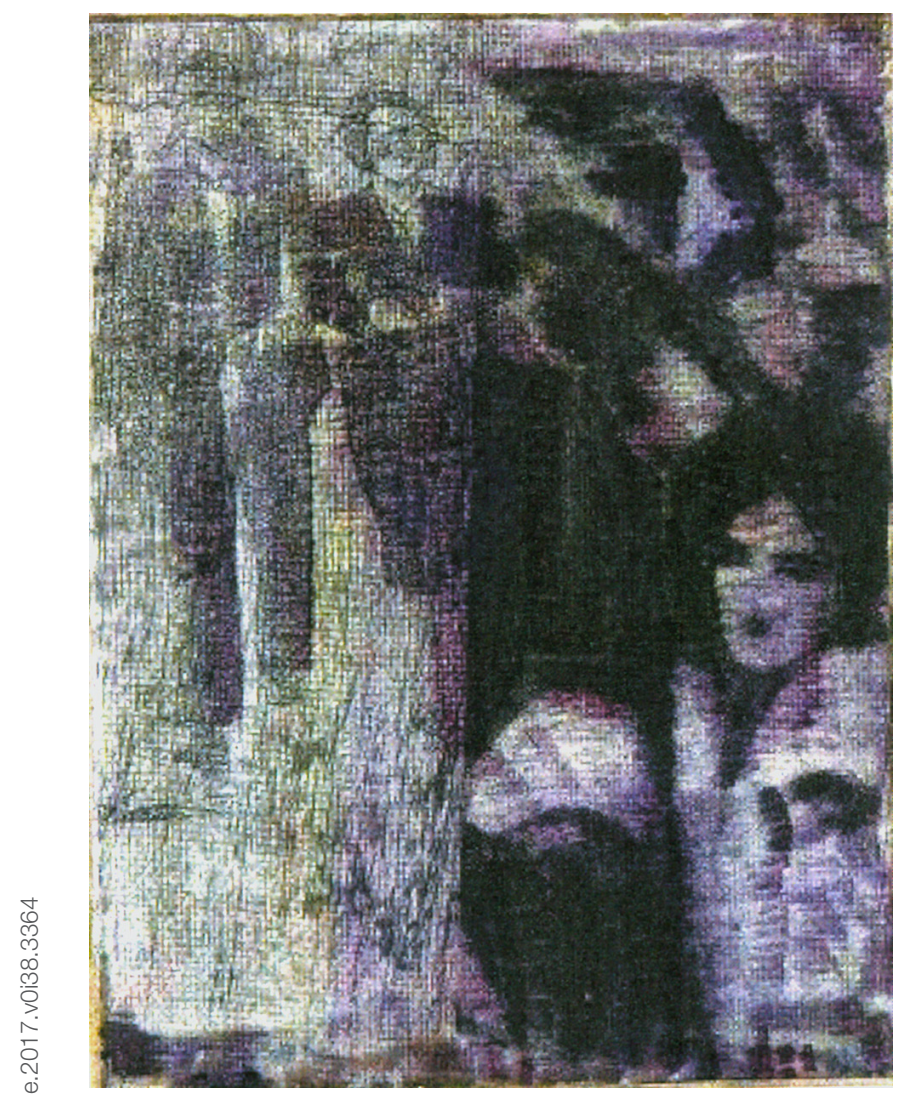

4. Bracha Ettinger, Eurydice n. 37, 1994-1998
L'artista Bracha Ettinger con l'opera Eurydice n. 37, 1994-1998 [4], esprime in maniera molto chiara la sua differenza in quanto donna e in quanto ebrea e fa uno chiaro riferimento post-Auschwitz. Con i suoi dipinti diventa una testimone, crea un linguaggio simbolico costituito di tracce e di segni, in grado di fornire un risarcimento a qualsiasi esclusione sia degli ebrei che delle donne offrendo un punto de vista nuovo e di rivisitare la nostra visione storica. Troviamo chiaramente la figura del padre e dalla madre dall'artista, cromaticamente l'opera è fatta su una gradazioni di grigio con acenti in viola ed ocra. La superficie pittorica è granulata divisa in due piani, il primo che corrisponde al volto di Eurydice ed il secondo piano dove si possono riconoscere i suoi genitori.

L'opera di Marcos López, Autopsia, dal 2005 [5], è una fotografia dipinta a mano. Quante autopsie si devono o possono fare in una guerra? II termine autopsia deriva del greco, aútóc/autós/ 'se stesso' y ö廿ıc /opsis/ 'observare', significa «vedere per se stesso». Nella immagine si osserva una donna sul tavolo con sette uomini intorno a lei. Un corpo ormai morto che ha perso la sua sensualità, con il torace cuscito con punti metallici e con una plastica a modo pannolino avvolgendo i genitali. Cromaticamente predominano i toni freddi: gli azzurri, grigi e verdi.

In una intervista, Marcos López chiarisce il significato e le influenze della immagine, la donna sul tavolo ripresenta la patria, sono le illusioni morte di un paese che no può essere ed infatti è una autopsia clandestina e perversa, per questa ragione il luogo è sporco e le finestre stanno sigillate

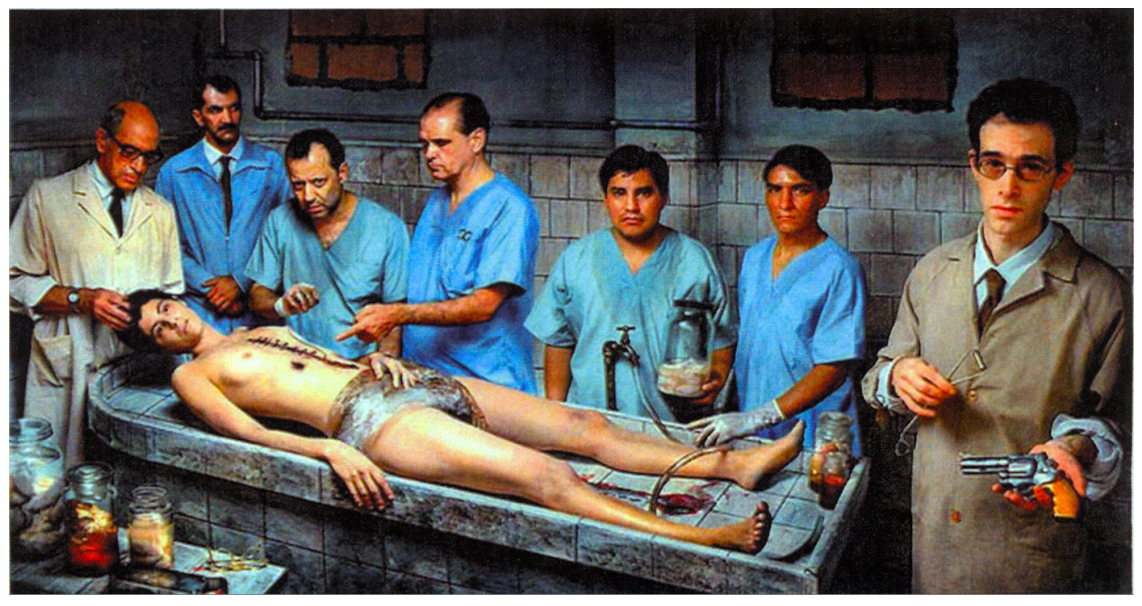

5. Marcos López. Autopsia, 2005. Fotografia a colore dipinta a mano. Ed. 5/5, Colección Galería El Museo, HABEAS CORPUS: que tengas [un] cuerpo [para exponer] Museo de Arte del Banco de la República, Bogotá 
per occultare i fatti. È il prodotto della unione di due idee o influenze, la fotografia del Che Guevara muerto en la Higuera (1967) e La lezione di anatomia dal Dr. Nicolaes Tulp. Lo stesso López, apare nella fotografia porta un revolver. Non è difficile immaginare che hanno cancellato le impronte digitali.

L'opera di Jenny Holzer, appartiene alla serie Anguish (Angoscia) dal 2010 [6], composta da diciassette pezzi, questa in particolare si intitola Lustmord (Lust = sessuale. Mord = assassinio), scava nella psique de le donne devastate per la guerra di Bosnia. In questa opera si osserva un tatuaggio sulla pelle in inglese: «I am awake in the place where women die». Holzer trascrive le parole delle vittime e dei suoi familiari che furono testimoni dell'orrore. I fatti furono incisi nella pelle delle persone che li subirono. La guerra durata più di tre anni causa centomila vittime, la maggioranza bosni. Fu la guerra più cruenta dalla Seconda Guerra Mondiale. Le tre parti contendenti -musulmani, serbi e croati- crearon o campi di concentramento come Omarska, KeratermeTrnopolje, dove furono torturate ed assassinate mille di persone. Gli abusi sessuali e le violazioni furono anche numerose durante la guerra.

L'opera di Von Hagens Card-playing corpses dal 2011 [7] è particolarmente abietta perché questi corpi sono cadaveri reali. Sono morti in vita, giocando alle carte. L'opera è un paradigma delle frontiere etiche, estetiche e culturali. Preserva il corpo umano per passare alla posterità, che alla fine è stato sempre un principio dell'arte, la gente si fa ritratti per sopravvivere al tempo, ad perpetuam (rei)memoriam; a perenne ricordo dell'avvenimento.

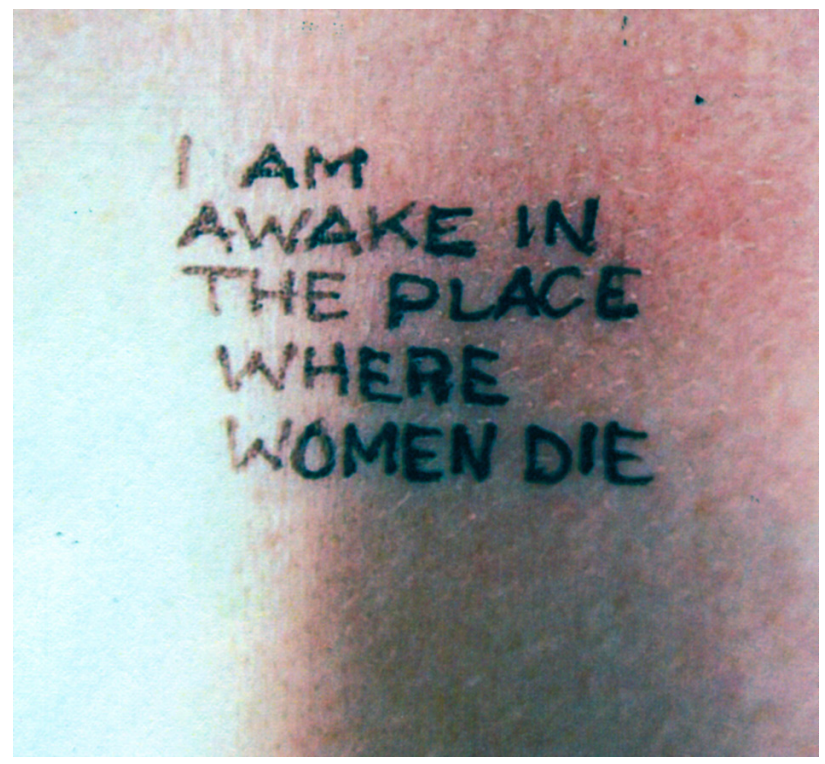

6. Jenny Holzer, Lustmord, detail-from-the-show-«Anguish», 2010. Museum of Contemporary Art Chicago

Un altro esempio lo troviamo nell' opera fotografica di Damien Garrick, Vision1 dal 2013 [8], questa composizione di un volto da un giovane in primo piano genera abiezione è un uomo morto, un cadavere, è un corpo -caduto- in parole di Julia Kristeva. È pieno di sangue ed ha espirato. Degli occhi azzurri che si perdono nel vuoto, lo sguardo che oltrepassa il campo visivo e si perde nell'abisso più profondo, la visione del trauma della morte. È una immagine anonima,
7. Gunther von Hagens, Cardplaying corpses, 2011, s/d Plastinarium in Guben, Germany

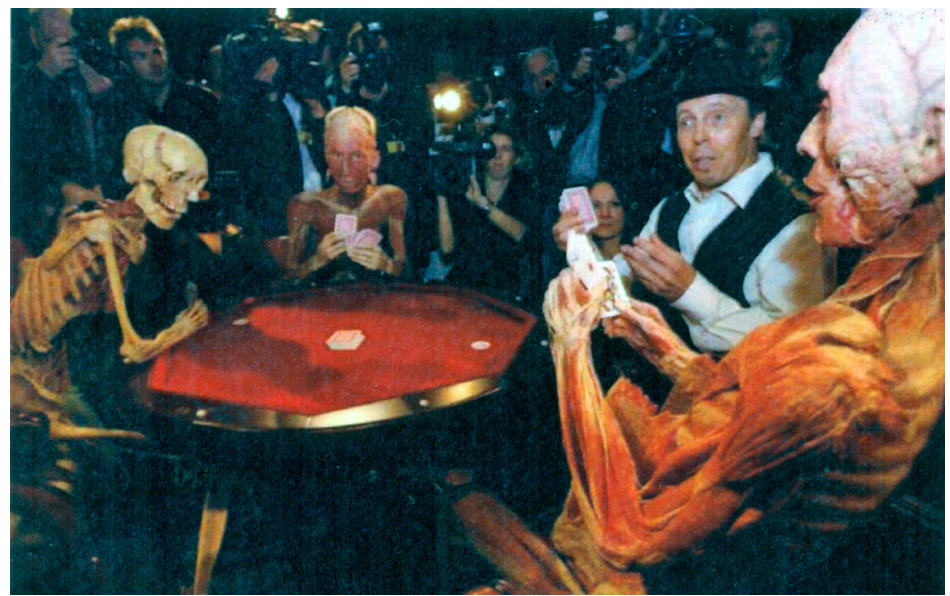




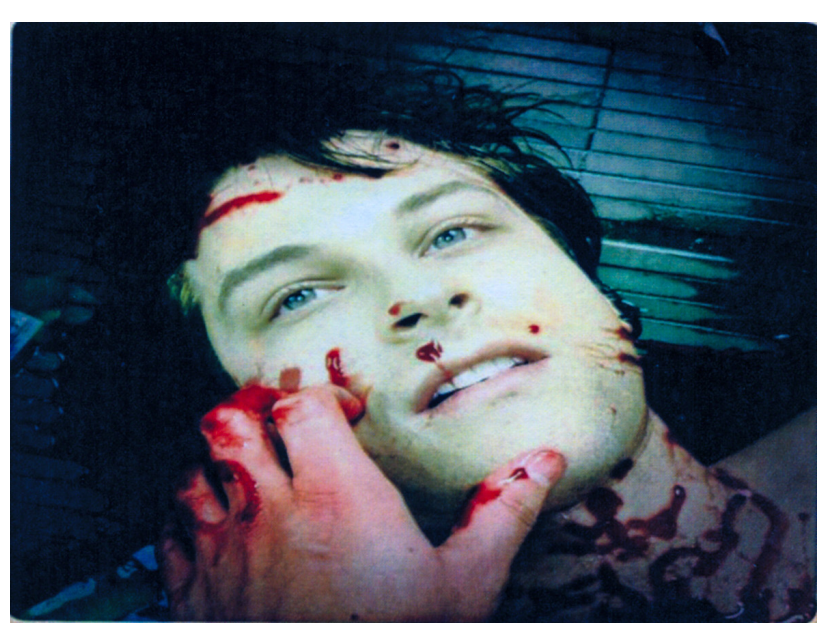

8. Damien Garrick. Vision1. 2013, Fotografia a colore, TAP Gallery, Darlinghurs

tetrica, inquietante che soltanto può essere guardata con freddezza dalla lontananza affettiva, che succederebbe se fosse un nostro conosciuto?

Concludendo con questioni molto problematiche del concetto di abiezione e, per tanto, da tenere in conto sono il grado di impatto, assimilazione e accettazione nella società, e la ragione per cui rivendichiamo l'esistenza di un linguaggio abietto, così come di una cultura abietta del corpo. L'obiettivo è quello di dimostrare che i nostri canoni comportamentali e di accettazione delle dottrine dogmatiche formano parte di una ideologia ed immagine collettiva, poiché essa si inserisce dentro un profilo etnografico, geoculturale, religioso, geopolitico e soprattutto perché, nella nostra società occidentale, essa ci porta a stabilire ciò che è abietto e ciò che non lo è.

Contrariamente a quello che si pensa, l' abiezione è sempre presente, e si confronta con la nostra condizione di cultura post-traumatica; è un dato oggettivo, per il peso di un' insostenibile memoria che provoca angoscia, la cui presenza spesso si nasconde. Tentiamo qui di far comprendere la contraddittorietà del fatto; solo considerando il concetto di abiezione come presente nella realtà, esso si può riconoscere ed affrontare ed è solo così che diventa testimonianza estetica nell'arte, si confronta con il trauma e si sublima. ॥ processo di riconoscimento porta ad accettare e, di conseguenza, a comprendere che il corpo e la società hanno bisogno dei rifiuti per potersi sostenere e per poter sopravvivere. L'abietto è ciclico, ripetitivo e paradossale.

\section{Bibliografia}

ARENDT, Hannah (1964), La banalità del male, Universale Economica Feltrinelli, Milano.

- (2006), L'umanità in tempi bui, Riflessioni su Lessing, Raffaello Cortina Editore, Milano.

- (2017), L'ebreo come paria, Una tradizione nascosta, Giuntina, Firenze.

BENNETT, Jill (2005), Empathic Vision Affect, Trauma and Contemporary Art, Stanford University Press, Stanford.

ETTINGER, Bracha (1999), Regard et Espace-de-bord matrixiels. Essais psychanalityques sur le feminin et le travail de l'art, La Lettre volée, Bruxelles.

- (2006), "Gaze-and-Touching the Not Enough Mother», in DE ZEGHER, C. (ed), Eva Hesse Drawing, Yale University Press, New York. HUBERMAN, G. Didi (2005), Immagini malgrado tutto, Raffaello Cortina Editore, Milano.

KRISTEVA, Julia (1980), Pouvoirs de l'horreur. Essai sur l'abjection, Editions du Seuil, Paris.

POLLOCK, Griselda (2013), After-affect, after-images: Trauma and aesthetic transformation in the virtual feminist museum, Manchester

University Press, Manchester.

SUBRIZI, Carla (2012), Azioni che cambiano il mondo. Donne, arte e politiche dello sguardo, Postmedia, Milano.

VIOLI, Patrizia (2014), Paesaggi della memoria: Il trauma, lo spazio, la storia, Bompiani, Milano.

TODOROV, Tzvetan (1992), Di fronte all'estremo. Vita e morte nei Lager e nei Gulag, Garzanti Elefanti, Milano.

WALSH, Maria (2013), Art and Psycoanalisis, Tauris, New York. 